\title{
Systemischer Lupus erythematodes
}

\section{Systemic Lupus Erythematosus}

Autoren

M. Aringer ${ }^{1}$, M. Schneider ${ }^{2}$

Institute

1 Medicine III, University Clinical Center Carl Gustav Carus at the TU Dresden, Dresden

2 Rheumatologie, Heinrich-Heine-Universität, Düsseldorf

Schlüsselwörter

Prednisolon, Hydroxychloroquin, Azathioprin, Methotrexat, Belimumab

Key words

prednisone, hydroxychloroquine, azathioprine, methotrexate, belimumab

Bibliografie

DOI https://doi.org/10.1055/s-0042-112363

Online-Publikation: 6.9.2016

Akt Rheumatol 2017; 42: 285-290

(c) Georg Thieme Verlag KG Stuttgart · New York ISSN 0341-051X

Korrespondenzadresse

Prof. Martin Aringer

Medicine III

University Clinical Center Carl Gustav Carus at the TU Dresden

Fetscherstraße 74

01307 Dresden

Tel.: + 49/351/458 4422, Fax: + 49/351/458 5801

Martin.Aringer@uniklinikum-dresden.de

\section{ZUSAMMENFASSUNG}

Die heutigen Therapieoptionen und -konzepte haben die Chancen von SLE-Patientinnen und -Patienten auf ein langes und gutes Leben mit Lupus deutlich verbessert. Gerade deshalb stellt die Erkrankung weiter eine relevante ärztliche Herausforderung dar. Die Breite der möglichen Organbeteiligung fordert den „Vollblut“-Internisten in jedem Rheumatologen heraus. Schübe, Infektionen und vaskuläre Ereignisse können kaum je längerfristig ausgeschlossen werden und benötigen von Patienten wie Ärzten ein hohes Maß an Aufmerksamkeit. Zusätzlich haben psychische Faktoren oft deutlichen Einfluss auf das Gesamtbild. Dennoch lassen sich für die meisten häufigen Situationen ähnlich wie bei der rheumatoiden Arthritis (RA) klare, einfache Regeln aufstellen. Diese sollen in der vorliegenden Übersicht skizziert werden.

\section{ABSTRACT}

Today's therapeutic options and concepts have greatly improved the chances of SLE patients to live a long and good life with lupus erythematosus. However, this is the very reason why SLE continues to be a considerable challenge for physicians. The broad spectrum of possible organ involvement requires every rheumatologist to be a "full-blooded" internal medicine specialist. Flares, infections and vascular events usually cannot be ruled out in the long run and necessitate vigilance both on the patient's and the physician's side. In addition, psychological factors often have a significant impact on the overall picture. Nevertheless, simple and straightforward rules can be formulated for most situations, as was done for rheumatoid arthritis (RA). This review aims to outline these rules.

\section{Diagnose SLE}

Die Klassifikationskriterien des American College of Rheumatology (ACR) [1, 2] haben über Jahrzehnte unser Verständnis des SLE wesentlich mitgeprägt. In einem Versuch, näher an auch diagnostisch verwertbare Charakteristika heranzukommen, haben die Kriterien der Systemic Lupus Cooperating Clinics (SLICC)-Gruppe die Sensitivität auf Kosten der Spezifität deutlich erhöht [3]. Aber beide Sets sind nicht für die Diagnose gemacht und sollten dafür auch nicht missbraucht werden - auch, weil sie zu viele Fehlermöglichkeiten haben. Obwohl momentan mit Hochdruck an noch besse- ren Klassifikationskriterien gearbeitet wird, wird sich an dieser Situation nichts Fundamentales ändern.

Was macht also die Diagnose SLE aus? Der Verdacht auf die Erkrankung sollte aufkommen, wenn typische Manifestationen (Haut, Arthritis, Niere,...) auftreten, wenn eine nicht erklärte Multi-Systemerkrankung vorliegt, oder Patienten unter länger andauernden Myalgien oder Arthralgien leiden und serologische Befunde (erhöhte BSG, Komplementverbrauch) vorliegen. Fast immer sind bei einem SLE die ANA positiv [4]. Allerdings ist hier unbedingt die Immunfluoreszenz auf Hep2-Zellen zu fordern [5]; Die ELISA-Verfahren sind weder in ihrer Sensitivität, noch in ihrer Spezifität vergleichbar. 
ANA sind allerdings nicht spezifisch (45\% Spezifität in der SLICCSLE-Kriterien-Kohorte (3). Sie sind ein gemeinsames Merkmal aller Kollagenosen, kommen aber auch bei der RA und anderen Autoimmunerkrankungen und Infektionen vor, und oft genug bei Gesunden. Besonders häufig führen positive ANA und Arthralgien im Rahmen einer Hashimoto-Thyroiditis zur Fehldiagnose SLE. Die Bestimmung der Schilddrüsen-Autoantikörper ist daher häufig sinnvoll. Andererseits sollten bei höhergradigem Verdacht auf einen SLE ENA (oder Anti-Ro-60-Antikörper) und Antikörper gegen doppelsträngige DNA (dsDNA) bestimmt werden, die die Spezifität des ANABefundes erhöhen [5].

Auch für die weiterführende serologische Diagnostik ist die Spezifität kritisch. Während Anti-Sm-Antikörper weitgehend SLE-spezifisch (99\%) sind, sind Anti-Ro-Antikörper beim Sjögren-Syndrom die Regel und kommen bei Systemischer Sklerose vor. Anti-dsDNAAntikörper sind recht spezifisch (96\%), wenn der Test spezifisch ist (CLIFT, RIA), aber Anti-dsDNA-Antikörper im ELISA sind leider meist sehr unspezifisch. Anti-U1RNP-Antikörper kommen isoliert bei der MCTD vor, sprechen aber in Kombination mit anderen Antikörpern für einen SLE. Anti-Phospholipid-Antikörper kommen etwas gleich häufig mit und ohne SLE vor - und gehen häufig mit positiven ANA einher ( $\triangleright$ Abb. 1).

Von den Organmanifestationen ist nur die histologisch gesicherte Lupus-Nephritis in der Regel spezifisch genug, um alleine darauf eine Diagnose aufzubauen [3]. Hautmanifestationen können auch isoliert als Haut-Lupus vorliegen, und haben zum Teil auch wichtige Differenzialdiagnosen (wie die Rosacea für die Rötung im Schmetterlingsareal). Wenn aber zumindest 2 unterschiedliche Organmanifestationen vorliegen, die annehmbar durch Autoantikörper oder Immunkomplexe bedingt sind (und für die es keine bessere Erklärung gibt), dann ist ein SLE wahrscheinlich. Ebenso reicht eine vermutlich immunkomplexbedingte Organmanifestation (Anti-CCP-negative Arthritis) in Kombination mit einem damit nicht zusammenhängenden positiven Antikörpertest (z. B. Lupus-Antikoagulans) meist für die Diagnose aus.

Die Differenzialdiagnosen beinhalten, neben verschiedensten Infektionen, hämatologischen Erkrankungen und manchmal auch genetisch bedingten Syndromen, vor allem andere Autoimmunerkrankungen. Manche davon können isoliert vorkommen (primäres APS, autoimmunhämolytische Anämie, Haut-Lupus), aber auch ein Teil des SLE-Spektrums sein.

\section{Basismaßnahmen}

Die Diagnose SLE triggert per se keine immunsuppressive Therapie, sondern indiziert einerseits Abklärungsschritte (insbesondere die Urinuntersuchung), und andererseits ein Set an Basismaßnahmen, das sich unter dem Akronym SASKIA zusammenfassen lässt [6]: Die Diagnose SLE sollte zum Einsatz von Antimalariamitteln (in der Regel 200 mg Hydroxychloroquin täglich), von Sonnenschutz (SPF 50 für alle Patienten mit UV-Empfindlichkeit/Hautbeteiligung),

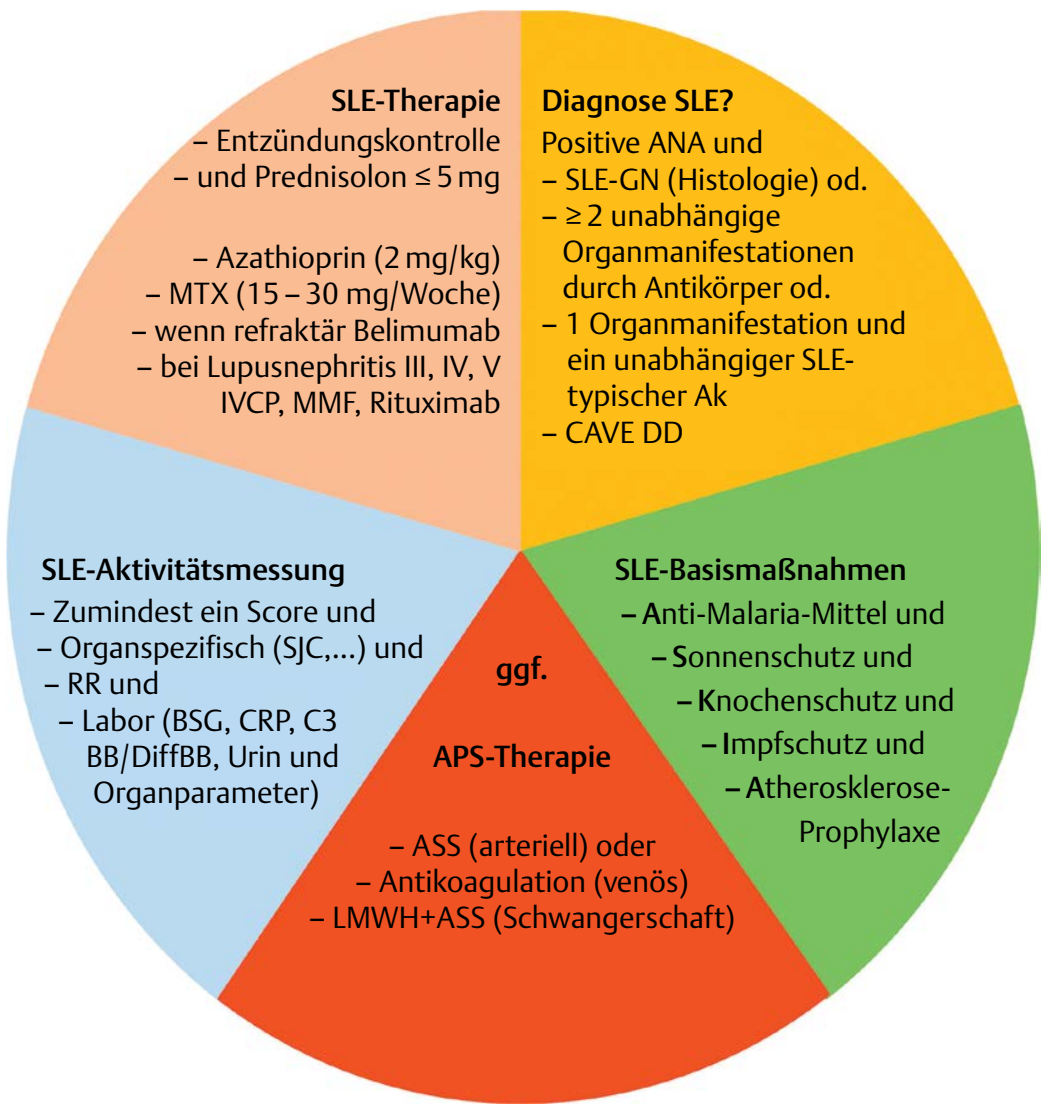

Abb. 1 Grundlagen des SLE-Managements von der Diagnose bis zur Therapie (im Uhrzeigersinn). 
Knochenschutz mit Vitamin D (20000 IU/Woche), optimalem Impfschutz und Atherosklerose-Risikoabschätzung führen. Diese Basismaßnahmen, vor allem auch Hydroxychloroquin, sollten unabhängig von zusätzlich notwendigen Schritten dauerhaft beibehalten werden.

Gerade beim Impfschutz haben die Daten der letzten 10 Jahre zu einem Umdenken geführt. Wurden früher Impfungen bei SLEPatienten kritisch gesehen, wurde inzwischen gezeigt, dass kaum je durch Impfungen relevante SLE-Schübe ausgelöst werden [7]. Hingegen sind SLE-Patienten massiv durch Infektionen gefährdet, auch durch Influenza und Pneumokokken, sodass diese Impfungen zusätzlich zum Standardprogramm für alle SLE-Patienten zu empfehlen sind [8].

Gefäßereignisse als Folge einer deutlich zu früh auftretenden Arteriosklerose werden zunehmend zu einer der häufigsten Todesursachen von SLE-Patienten [9]. Gerade jüngere Patientinnen haben ein dramatisch erhöhtes Gefäßrisiko [10]. Daher müssen einerseits die SLE-Aktivität kontrolliert und andererseits die längerfristige höher dosierte Glukokortikoidgabe ( $>5 \mathrm{mg}$ täglich) vermieden werden, andererseits aber die konventionellen Risikofaktoren (Nikotin, RR, Cholesterin...) erhoben und gegebenenfalls beeinflusst werden. Sowohl für die Impfung als auch für das Atherosklerose-Risikomanagement ist die Zusammenarbeit mit dem Hausarzt entscheidend.

\section{Sonderfall sekundäres Anti-Phospholipid-Syndrom}

Bei einem relevanten Teil der SLE-Patienten können Antikörper gegen Phospholipide gefunden werden, und bei einer Subgruppe dieser Patienten liegt ein (dann sekundäres) Anti-Phospholipid-Syndrom vor. Anti-Phospholipid-Antikörper sind heute nicht ausreichend immunologisch beeinflussbar. Alle Patienten mit venösem Ereignis müssen daher dauerhaft (zumindest bis zum Vorliegen von Studien mit neuen oralen Antikoagulanzien mit Coumadinen) antikoaguliert, alle mit arteriellem Ereignis zumindest mit ASS behandelt werden [11]. In Schwangerschaften von Patientinnen mit Anti-Phospholipid-Syndrom kommen niedermolekulare Heparine in Kombination mit ASS zum Einsatz [12]. Liegen Antikörper bei SLE ohne Klinik vor, ist zusätzlich zu Hydroxychloroquin ASS zu erwägen, das zumindest für die noch kritischeren arteriellen Ereignisse eine wirksame Prophylaxe darstellt [13].

\section{SLE-Aktivität}

Bei einer komplexen Autoimmunerkrankung wie dem SLE müssen für die Gesamtbeurteilung der Aktivität Überblicksinstrumente und organbezogene Parameter kombiniert werden [8]. Bei Patienten ohne Nierenbeteiligung wird der Urinstreifen, bei Patienten ohne Gelenkbeteiligung die Frage nach Gelenkschmerzen ausreichen. Bei Nierenbeteiligung wird hingegen die regelmäßige Quantifizierung der Proteinurie, bei Gelenkbeteiligung der Gelenkbefund (wie bei der RA) erforderlich.

Um nicht relevante Aspekte zu übersehen, wird die Verwendung zumindest eines standardisierten Aktivitätsscores (ECLAM, SLEDAI, SIS,...) empfohlen. Dabei kommt es aber nicht nur auf den Wert, sondern auf den Gesamtüberblick an. Die Blutdruckmessung sollte auch bei gerade asymptomatischen SLE-Patienten jedes Mal erfolgen. Die Schmerz-Skala kann unter anderem schon Hinweise auf ein nicht selten vorhandenes (sekundäres) Fibromyalgie-Syndrom geben.
Die Laborkontrollen sollten neben BSG und CRP die Komplementkomponenten C3 und C4, Blutbild (und Differenzialblutbild), die wesentlichen Organparameter und den Urin(streifen)befund umfassen. Deutlich erhöhte CRP-Werte sprechen in erster Linie für bakterielle Infektionen, und nicht primär für Krankheitsaktivität, kommen aber bei Lupusarthritis und Serositis vor. Von den Autoantikörpern korrelieren ANA und ENA nicht mit der Erkrankungsaktivität; eine regelmäßige Wiederholung ist daher entbehrlich. Ebenso ist eine regelmäßige Wiederholung von Anti-PhospholipidAntikörpern in der Regel nicht sinnvoll. Hingegen können Anti-dsDNA-, Anti-Histon- und Anti-Chromatin/Nukleosomen-Antikörper immunologische Aktivität anzeigen.

\section{Standardtherapie}

Hydroxychloroquin sollte jeder SLE-Patient ohne Kontraindikationen einnehmen. Darüber hinaus medikamentös sollten nach derzeitigem Stand hingegen nie rein immunologische Befunde, sondern nur relevante Organsymptome und eine annehmbar durch SLE-Aktivität bedingte Allgemeinsymptomatik behandelt werden. Im Zweifel müssen Infektionen unbedingt zuerst ausgeschlossen und eventuell auf Verdacht behandelt werden, insbesondere bei hohem CRP [14].

Glukokortikoide stellen nach wie vor den Standard in der raschen Anbehandlung von SLE-Symptomen dar. In der Regel reichen dafür $0,5 \mathrm{mg} / \mathrm{kg}$ täglich aus. Aufgrund der hohen Langzeittoxizität und insbesondere des negativen Einflusses auf Infektionen [15] und kardiovaskuläres Risiko [16, 17] sollte die Dosis nicht längerfristig auf $>5 \mathrm{mg}$ Prednisolonäquivalent täglich belassen werden. Wenn die Krankheitsaktivität mehr erforderlich macht, sollten stattdessen Methotrexat (MTX), Azathioprin oder Belimumab eingesetzt werden.

Auch wenn es für den SLE nicht zugelassen ist, ist MTX beim SLE wie bei der RA eine auch langfristig sehr sichere Therapie, so lange die Nierenfunktion erhalten ist [18]. Insbesondere bei entzündlichen Manifestationen des Muskuloskeletalsystems, der Haut und der Gefäße ist MTX in einer wöchentlichen Dosis von 15-25 mg eine meist sehr gute Wahl.

Für Azathioprin ist die Datenlage außerhalb der Erhaltungstherapie der Lupusnephritis formal schlechter als für MTX [19]. Azathioprin ist aber seit Jahrzehnten eine Standardtherapie. Die Substanz darf nicht mit Allopurinol oder Febuxostat kombiniert werden und sollte anfangs vorsichtig begonnen und engmaschig überwacht werden, um schwere Blutbildveränderungen oder eine Azathioprin-Hepatitis rechtzeitig zu erkennen. Zur Kontrolle der Aktivität werden in der Regel $2 \mathrm{mg} / \mathrm{kg}$ täglich empfohlen, längerfristig ist eine Reduktion aber oft möglich.

Wenn keine lebensgefährliche Organbeteiligung vorliegt und die Erkrankung durch Azathioprin und gegebenenfalls MTX nicht so weit kontrolliert werden kann, dass die Prednisolondosis konstant auf $5 \mathrm{mg}$ oder weniger bleibt, ist Belimumab der zugelassene nächste Therapieschritt. Dieses erste zugelassene SLE-Biologikum ist keine schnell wirksame Substanz, kontrolliert aber im Laufe von 3-6 Monate viele entzündliche Organprobleme und kann relativ rasch eine immunologisch mediierte Fatigue bessern [20,21]. Sicherheitsrelevant sind vor allem seltene gefährliche Infusionsreaktionen. Dieses Problem wird vermutlich mit der Einführung der subkutanen Variante gelöst sein. 


\section{Nephritis und ZNS-Beteiligung}

Standardtherapie für die schwere Lupusnephritis ist weiterhin Cyclophosphamid, allerdings in der reduzierten Euro-Lupus-Dosis von insgesamt $3 \mathrm{~g}(6 \times 500 \mathrm{mg})$ Gesamtdosis [22, 23]. Darunter ist das Risiko des irreversiblen Ovarialversagens offenbar verringert. Im Anschluss wird standardmäßig Azathioprin (2 mg/ $\mathrm{kg}$ ) als Erhaltungstherapie eingesetzt.

Alternativ gibt es für Mycophenolat-Mofetil (MMF) schlüssige Daten, dass diese für den SLE nicht zugelassene Substanz in der Induktionsphase in einer Dosis von $3 \mathrm{~g}$ täglich gleich gut wie (bei Afrikanern afrikanischer Herkunft sogar besser als) Cyclophosphamid $[24,25]$ und in der Erhaltung in einer Dosis von $2 \mathrm{~g}$ täglich besser als Azathioprin wirkt [26-28]. MMF schädigt zwar die Eierstockfunktion nicht dauerhaft, ist aber klar teratogen. In einem aktuellen Rote-Hand-Brief wurden die Sicherheitsmaßnahmen zur Verhütung einer Schwangerschaft erhöht. Für Mycophenolsäure (MPA) sind nunmehr auch gewisse SLE-Daten vorhanden [29]; ein relevanter Unterschied zwischen 500 mg MMF und 360 mg MPA ist nicht zu erwarten. MPA hat Vorteile, wenn MMF zu abdominellen Beschwerden führt.

Etwas anders stellt sich die Situation für die schwere ZNS-Beteiligung dar. Zunächst ist darauf hinzuweisen, dass der Großteil der vaskulären ZNS-Ereignisse durch Anti-Phospholipid-Syndrom oder Atherosklerose bedingt sind [30]. Für den eher seltenen Fall einer ZNS-Vaskulitis ist ein Effekt von Cyclophosphamid nachgewiesen. Die Euro-Lupus-Dosis wurde allerdings nie formal untersucht. Die Datenlage spricht bei der Vaskulitis eher dafür, dass Azathioprin dem MMF überlegen ist.

Sowohl bei therapierefraktärer schwerer Lupusnephritis als auch bei therapierefraktärer ZNS-Vaskulitis ist die off label-Therapie mit Rituximab international üblicher Standard [23, 31]. Darüber hinaus wird Rituximab auch dann oft erfolgreich eingesetzt, wenn andere vital bedrohliche Organmanifestationen, insbesondere eine schwere Vaskulitis, eine Lupuspneumonitis oder interstitielle Lungenbeteiligung, eine Lupus-Myokarditis oder bedrohliche therapierefraktäre hämatologische Manifestationen nicht kontrollierbar scheinen [31].

Insbesondere bei schwerster, therapierefraktärer Multiorganbeteiligung kommen auch gegen langlebige Plasmazellen gerichtete Therapien, nämlich die off label-Gabe des Proteasom-Inhibitors Bortezomib [32] und die leider in dieser Situation mit einer hohen Letalität behaftete autologe Stammzelltherapie [33] in Frage.

\section{Problematik off label-Therapie}

Leider sind die meisten annehmbar erfolgreichen Therapien bisher nicht für den SLE zugelassen. De facto gilt das sogar für MTX, auch wenn diese Therapie bisher aufgrund der geringen Kosten nie zu Erstattungsproblemen geführt hat. Für vital bedrohliche Situationen, in denen die zugelassene Standardtherapie ausgereizt ist, ist ein rationaler off label-Einsatz rechtlich an sich klar gedeckt und bedarf lediglich eines erhöhten Aufklärungsaufwands, der dokumentiert werden sollte. In der Realität ist es aber leider nicht immer einfach, MDK-Kollegen und Krankenkassen von der vital bedrohlichen Situation und dem Fehlen adäquater zugelassener Therapien zu überzeugen. Gerade bei seltenen Problemen im Rahmen selte- ner Erkrankungen fehlt auf Kostenträgerseite häufig die medizinische Expertise, da gilt es Überzeugungsarbeit zu leisten.

Noch komplexer ist die Situation bei schwerwiegenden, aber nicht vital bedrohlichen Problemen. Hier hat der Gesetzgeber bisher die Rahmenbedingungen nicht ausreichend klar festgelegt. Die Konsequenz sind viele rechtliche Auseinandersetzungen und annehmbar eine medizinische Unterversorgung von Patienten mit therapierefraktären Verläufen seltener Erkrankungen. Es erscheint zum Beispiel widersinnig, ein für die RA routinemäßig verwendetes Biologikum bei einer schweren, therapierefraktären Lupus-Arthritis mit vergleichbaren Folgen für den Patienten nicht einsetzen zu können, wenn die zugelassene Therapie nicht ausreichend wirkt. Zum Beispiel gäbe es für die off label-Gabe des IL-6-Rezeptor-Blockers Tocilizumab durchaus Daten [34], die für eine Wirksamkeit sprechen, aber kein Interesse des Herstellers an weiteren Studien. Für Abatacept gibt es ebenfalls gewisse Daten [35]; eine weitere klinische Prüfung wird durchgeführt. Und sogar Etanercept war in einer großen Fallserie aus Barcelona wirksam und erstaunlich sicher [36].

\section{Neue Therapieansätze in Studien}

Aufgrund einer hohen Zahl neuer Ansätze in klinischer Prüfung (www.clinicaltrials.gov) ist zu hoffen, dass sich die Situation für SLEPatienten mit therapierefraktären Problemen sukzessive entspannen wird. Das wird aber noch einige Jahre benötigen. Unter den neuen Ansätzen erscheint der Anti-Typ I-Interferon-Antikörper Anifrolumab besonders interessant, der sich Medimmune-intern gegen den Anti-Interferon- $\alpha$-Antikörper Sifalimumab durchgesetzt hat. Zumindest in Abstractform sehen die Ergebnisse sehr erfreulich aus [37]; besondere Sicherheitssignale sind bisher nicht zu erkennen. Ebenso werden mehrere Moleküle gegen IL-6 oder den IL6-Rezeptor getestet, wobei zumindest zweitere ebenfalls eine gute Chance haben. In der Kombination Interferon-Rezeptor und IL6-Rezeptor, die beide wesentlich auf die Januskinase-1 (Jak1) angewiesen sind, erscheint die Jak-Blockade durchaus interessant. Tatsächlich werden sowohl Baricitinib als auch Tofacitinib beim SLE geprüft. Ebenso wird mit Blisibimod ein zusätzlicher Anti-BlyS/ BAFF-Ansatz getestet.

Atacicept, das sowohl BLyS/BAFF als auch das Schwesterzytokin APRIL blockiert, führt offenbar regelhaft zur IgG-Verminderung. Weil das voraussichtlich bedeutet, dass die Substanz auch langlebige Plasmazellen beeinflusst, könnte Atacicept eher für ein vielleicht kuratives Szenario interessant sein. In dieser Richtung werden sonst Proteasominhibitoren und der Anti-CD38-Antikörper Daratuzumab getestet. Gegen B-Zellen richten sich neben Rituximab und dem neuen Anti-CD20-Antikörper Obinutuzumab, ein AntiCD19-Antikörper und ein Inhibitor der BTK (Brutons Tyrosinkinase).

In der Kostimulationsblockade werden neben Abatacept der Anti-CD28-Antikörper Lulizumab und Ansätze gegen die CD40CD40L-Kostimulation geprüft. Weitere T-Zell-Ansätze beinhalten den Anti-CD28-Superagonisten TAB08 und die niedrigdosierte Gabe von IL-2. Gegen Immunkomplexe richtet sich RSLV-132, ein Fc-Rezeptor-Nuklease-Hybridmolekül. Und schließlich wird auch das aus dem U1 70kD Protein stammende Serin-phosphorylierte Peptid Rigerimod weiter für die spezifische Immuntherapie des SLE 
getestet. Es ist zu hoffen, dass wenigstens einige dieser Ansätze in den nächsten Jahren zu Zulassungen führen werden.

\section{Zusammenfassung}

Neue Substanzen werden vermutlich in den nächsten Jahren die SLE-Therapie weiter verbessern, und neue SLE-Klassifikationskriterien sollten Fortschritte in der richtigen Einordnung der Erkrankung bringen. Die Strategie bei Diagnose, Basismaßnahmen und Aktivitätseinschätzung kann durch bessere Biomarker und eine klare Ausrichtung auf Remission optimiert werden. Auch wenn wir mehr Werkzeuge haben werden, um das auch sicher zu erreichen, werden weiter das Stoppen entzündlicher Aktivität und das Vermeiden von Prednisolondosen > 5 mg täglich im Zentrum stehen.

\section{Interessenkonflikt}

M. Aringer: Teilnahme an Advisory Boards von und/oder Vorträge für Astra Zeneca, BMS, Chugai, GSK, Lilly, Pfizer, Roche. M. Schneider: Teilnahme an Advisory Boards von und/oder Vorträge für Abbvie, Astra Zeneca, BMS, Chugai, GSK, Lilly, Medimmune, MSD, Novartis, Pfizer, Roche, Suppremol, UCB Forschungsförderung Abbvie, GSK und UCB.

\section{Literatur}

[1] Tan EM, Cohen AS, Fries JF et al. The 1982 revised criteria for the classification of systemic lupus erythematosus. Arthritis Rheum 1982; 25: $1271-1277$

[2] Hochberg MC. Updating the American College of Rheumatology revised criteria for the classification of systemic lupus erythematosus. Arthritis Rheum 1997; 40: 1725

[3] Petri M, Orbai AM, Alarcon GS et al. Derivation and validation of systemic lupus international collaborating clinics classification criteria for systemic lupus erythematosus. Arthritis Rheum 2012; 64: 2677-2686

[4] Leuchten N, Brinks R, Hoyer A et al. Anti-nuclear antibodies have high sensitivity for systemic lupus erythematosus: results of a systematic literature review and meta-regression of diagnostic data. Arthritis Rheumatol 2015; 67: (Suppl 10): (abstract 774). Ref Type: Abstract

[5] Agmon-Levin N, Damoiseaux J, Kallenberg C et al. International recommendations for the assessment of autoantibodies to cellular antigens referred to as anti-nuclear antibodies. Ann Rheum Dis 2014; 73: $17-23$

[6] Aringer M, Schneider M. Recipes systemic lupus erythematosus. Z Rheumatol 2014

[7] Touma Z, Gladman DD, Urowitz MB. Vaccination and auto-immune rheumatic diseases: lessons learnt from the 2009 H1N1 influenza virus vaccination campaign. Curr Opin Rheumatol 2013; 25: 164-170

[8] Mosca M, Tani C, Aringer M et al. European League Against Rheumatism recommendations for monitoring patients with systemic lupus erythematosus in clinical practice and in observational studies. Ann Rheum Dis 2010; 69: 1269-1274

[9] Yurkovich M, Vostretsova K, Chen W et al. Overall and cause-specific mortality in patients with systemic lupus erythematosus: a meta-analysis of observational studies. Arthritis Care Res (Hoboken) 2014; 66: 608-616
[10] Roman M], Shanker BA, Davis A et al. Prevalence and correlates of accelerated atherosclerosis in systemic lupus erythematosus. $N$ Engl J Med 2003; 349: 2399-2406

[11] Specker C. Antiphospholipid syndrome. Z Rheumatol 2015; 74: 191-198

[12] Fischer-Betz R, Spathling-Mestekemper S. Pregnancy and inflammatory rheumatic diseases. Z Rheumatol 2013; 72: 669-682

[13] Arnaud L, Mathian A, Ruffatti A et al. Efficacy of aspirin for the primary prevention of thrombosis in patients with antiphospholipid antibodies: an international and collaborative meta-analysis. Autoimmun Rev 2014; 13: 281-291

[14] Kim HA, Jeon JY, An JM et al. C-reactive protein is a more sensitive and specific marker for diagnosing bacterial infections in systemic lupus erythematosus compared to $\mathrm{S100A8/A9}$ and procalcitonin. J Rheumatol 2012; 39: 728-734

[15] Listing J, Gerhold K, Zink A. The risk of infections associated with rheumatoid arthritis, with its comorbidity and treatment. Rheumatology (Oxford) 2013; 52: 53-61

[16] Ajeganova S, Svensson B, Hafstrom I. Low-dose prednisolone treatment of early rheumatoid arthritis and late cardiovascular outcome and survival: 10-year follow-up of a 2-year randomised trial. BMJ Open 2014; 4: e004259

[17] Roubille $C$, Richer $V$, Starnino T et al. The effects of tumour necrosis factor inhibitors, methotrexate, non-steroidal anti-inflammatory drugs and corticosteroids on cardiovascular events in rheumatoid arthritis, psoriasis and psoriatic arthritis: a systematic review and meta-analysis. Ann Rheum Dis 2015; 74: 480-489

[18] Winzer M, Aringer M. Use of methotrexate in patients with systemic lupus erythematosus and primary Sjogren's syndrome. Clin Exp Rheumatol 2010; 28: (Suppl 61): S156-S159

[19] Bertsias G, loannidis JP, Boletis J et al. EULAR recommendations for the management of systemic lupus erythematosus. Report of a Task Force of the EULAR Standing Committee for International Clinical Studies Including Therapeutics. Ann Rheum Dis 2008; 67: 195-205

[20] Furie R, Petri M, Zamani O et al. A phase III, randomized, placebocontrolled study of belimumab, a monoclonal antibody that inhibits $B$ lymphocyte stimulator, in patients with systemic lupus erythematosus. Arthritis Rheum 2011; 63: 3918-3930

[21] Navarra SV, Guzman RM, Gallacher AE et al. Efficacy and safety of belimumab in patients with active systemic lupus erythematosus: a randomised, placebo-controlled, phase 3 trial. Lancet 2011; 377: 721-731

[22] Houssiau FA, Vasconcelos C, D'Cruz D et al. The 10-year follow-up data of the Euro-Lupus Nephritis Trial comparing low-dose and high-dose intravenous cyclophosphamide. Ann Rheum Dis 2010; 69: 61-64

[23] Bertsias GK, Tektonidou M, Amoura Z et al. Joint European League Against Rheumatism and European Renal Association-European Dialysis and Transplant Association (EULAR/ERA-EDTA) recommendations for the management of adult and paediatric lupus nephritis. Ann Rheum Dis 2012; 71: 1771-1782

[24] Ginzler EM, Dooley MA, Aranow C et al. Mycophenolate mofetil or intravenous cyclophosphamide for lupus nephritis. N Engl J Med 2005; 353: 2219-2228

[25] Appel GB, Contreras G, Dooley MA et al. Mycophenolate mofetil versus cyclophosphamide for induction treatment of lupus nephritis. J Am Soc Nephrol 2009; 20: 1103-1112

[26] Dooley MA, Jayne D, Ginzler EM et al. Mycophenolate versus azathioprine as maintenance therapy for lupus nephritis. N Engl J Med 2011; 365: 1886-1895 
[27] Houssiau FA, D'Cruz D, Sangle S et al. Azathioprine versus mycophenolate mofetil for long-term immunosuppression in lupus nephritis: results from the MAINTAIN Nephritis Trial. Ann Rheum Dis 2010; 69: 2083-2089

[28] Aringer M, Fischer-Betz R, Hiepe F. Statement on the use of mycophenolate mofetil for systemic lupus erythematosus. Z Rheumatol 2013; 72: 575-580

[29] Jones RB, Walsh M, Chaudhry AN et al. Randomized trial of entericcoated mycophenolate sodium versus mycophenolate mofetil in multi-system autoimmune disease. Clin Kidney J 2014; 7: 562-568

[30] Bertsias GK, loannidis JP, Aringer $M$ et al. EULAR recommendations for the management of systemic lupus erythematosus with neuropsychiatric manifestations: report of a task force of the EULAR standing committee for clinical affairs. Ann Rheum Dis 2010; 69: 2074-2082

[31] Aringer M, Burkhardt H, Burmester GR et al. Current state of evidence on „off-label“ therapeutic options for systemic lupus erythematosus, including biological immunosuppressive agents, in Germany, Austria and Switzerland - a consensus report. Lupus 2012; 21: 386-401

[32] Alexander T, Sarfert R, Klotsche J et al. The proteasome inhibitior bortezomib depletes plasma cells and ameliorates clinical manifestations of refractory systemic lupus erythematosus. Ann Rheum Dis 2015; 74: 1474-1478
[33] Alexander T, Thiel A, Rosen O et al. Depletion of autoreactive immunologic memory followed by autologous hematopoietic stem cell transplantation in patients with refractory SLE induces long-term remission through de novo generation of a juvenile and tolerant immune system. Blood 2009; 113: 214-223

[34] Illei GG, Shirota Y, Yarboro CH et al. Tocilizumab in systemic lupus erythematosus: Data on safety, preliminary efficacy, and impact on circulating plasma cells from an open-label phase I dosage-escalation study. Arthritis Rheum 2010; 62: 542-552

[35] Merrill JT, Burgos-Vargas R, Westhovens R et al. The efficacy and safety of abatacept in patients with non-life-threatening manifestations of systemic lupus erythematosus: results of a twelve-month, multicenter, exploratory, phase llb, randomized, double-blind, placebo-controlled trial. Arthritis Rheum 2010; 62: 3077-3087

[36] Cortes-Hernandez ], Egri N, Vilardell-Tarres M et al. Etanercept in refractory lupus arthritis: An observational study. Semin Arthritis Rheum 2015; 44: 672-679

[37] Furie R, Merrill JT, Werth VP et al. Anifrolumab, an anti-interferon alpha receptor monoclonal antibody, in moderate to severe systemic lupus eyrthematosus (SLE). Arthritis Rheumatol 2015; 67 (Suppl 10): \#3223 Ref Type: Abstract 